\title{
Transconjunctival orbital decompression in Graves' ophthalmopathy: lateral wall approach ab interno
}

\author{
Dion A Paridaens, Karin Verhoeff, Dimitri Bouwens, Willem A van den Bosch
}

\begin{abstract}
Aims-A modified surgical technique is described to perform a one, two, or three wall orbital decompression in patients with Graves' ophthalmopathy. Methods-The lateral wall was approached ab interno through a "swinging eyelid" approach (lateral canthotomy and lower fornix incision) and an extended periosteum incision along the inferior and lateral orbital margin. In addition, the orbital floor and medial wall were removed when indicated. To minimise the incidence of iatrogenic diplopia, the lateral and medial walls were used as the first surfaces of decompression, leaving the "medial orbital strut" intact. During 1998, this technique was used in a consecutive series of 19 patients ( 35 orbits) with compressive optic neuropathy (six patients), severe exposure keratopathy (one patient), or disfiguring/congestive Graves' ophthalmopathy (12 patients).
\end{abstract}

Results-The preoperative Hertel value (35 eyes) was on average $25 \mathrm{~mm}$ (range 19-31 $\mathrm{mm}$ ). The mean proptosis reduction at 2 months after surgery was $5.5 \mathrm{~mm}$ (range 3-7 mm). Of the total group of 19 patients, iatrogenic diplopia occurred in two $(12.5 \%)$ of 16 patients who had no preoperative diplopia or only when tired. The three other patients with continuous preoperative diplopia showed no improvement of double vision after orbital decompression, even when the ocular motility (ductions) had improved. In the total group, there was no significant change of ductions in any direction at 2 months after surgery. All six patients with recent onset compressive optic neuropathy showed improvement of visual acuity after surgery. No visual deterioration related to surgery was observed in this study. A high satisfaction score (mean 8.2 on a scale of 1 to 10) was noted following the operation.

Conclusion-This versatile procedure is safe and efficacious, patient and cost friendly. Advantages are the low incidence of induced diplopia and periorbital hypaesthesia, the hidden and small incision, the minimal surgical trauma to the temporalis muscle, and fast patient recovery. The main disadvantage is the limited exposure of the posterior medial and lateral wall.

(Br f Ophthalmol 2000;84:775-781)
For many years orbital decompression has been a mainstay in the treatment of Graves' ophthalmopathy, although there has been a continuing search for alternative methods. Originally, orbital decompression was performed for compressive optic neuropathy and severe proptosis with keratopathy. ${ }^{1}$ Evolving surgical techniques with lesser side effects, and an increasing demand of patients towards cosmetic rehabilitation, have led to the use of orbital decompression for disfiguring exophthalmos.

Orbital decompression results from removal of bony orbital walls, followed by herniation of orbital contents into the periorbital spaces. This process is augmented by opening of the periorbita and disruption of orbital fibrotic bands and septae, especially in case of extensive orbital (post)-inflammatory fibrosis. Alternatively, orbital decompression may be achieved by removal of only orbital fat. ${ }^{2}$ In "bony" decompression, reduction of proptosis mainly depends on the number of orbital walls removed, the extent to which they are removed, and the anatomy of the bony orbit and periorbital spaces. Most of the reports on this issue ${ }^{3-6}$ describe different techniques to expose the orbital walls. The majority of patients can be adequately treated with a one, two, or three wall decompression. Only extreme cases of Graves' ophthalmopathy require four wall orbital decompression. ${ }^{7}$

A transconjunctival approach to the orbit, using a lateral canthal and inferior fornix incision ("swinging eyelid"), was first described by $\mathrm{McCord}^{8}$ in 1981 and recently popularised by Rootman and co-workers. ${ }^{9}$ This versatile technique provides adequate exposure of the medial orbital wall and the floor. In addition, access to the lateral orbital wall can be achieved through an extended lateral canthotomy (Burke's incision) and temporary removal of the lateral orbital rim, as in a lateral orbitotomy. Since removal of the lateral orbital rim and dissection of the temporalis muscle origin from the lateral wall inflict morbidity, we modified this technique by approaching the lateral wall $\mathrm{ab}$ interno, leaving the rim and the major part of the temporalis muscle and its blood supply intact. In this paper, we describe the results of this method in 19 consecutive patients (35 orbits) with Graves' ophthalmopathy who underwent a two or three wall orbital decompression. 


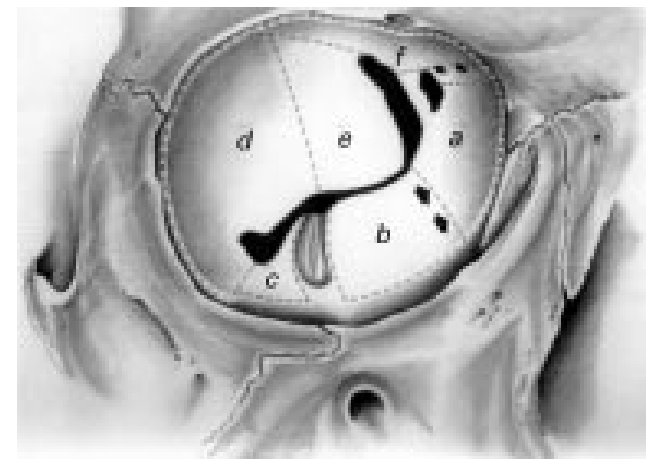

Figure 1 Anterior view of the right bony orbit. The four orbital walls can be selectively removed for orbital

decompression. The following walls are identified: the medial wall (a), the orbital floor, divided by the infraorbital nerve into a medial part (b), and a lateral part (c), the anterolateral wall (d), the posterolateral wall (e), and the orbital roof $(f)$. The bony junction between ( $a$ ) and $(b)$, the "orbital strut", $i$ s arrowed.

\section{Patients, methods, and surgical technique}

Between January 98 and January 1999, 19 patients (35 orbits) with Graves' ophthalmopathy underwent transconjunctival orbital decompression, using the technique described below. Fifteen patients were female and four were male. Their age ranged from 23 to 78 years (mean 48 years). The indications for surgery were compressive optic neuropathy in six patients, severe exposure keratopathy in one patient, and cosmetic rehabilitation in 12 patients. Compressive optic neuropathy was diagnosed when there was a decrease in visual acuity, visual field defects, abnormal visual evoked potentials (VEPs), and/or optic disc oedema. The desired amount of proptosis reduction was estimated preoperatively, and used as an indication for the extent of orbital bone removal and the number of orbital walls to be resected.

Eighteen patients (33 orbits) underwent a three wall decompression, and one patient (two orbits) a two wall decompression (lateral and medial wall) (Fig 1). To spare the infraorbital nerve and to reduce the risk of a "dropped bulbus" (hypoglobus), ${ }^{10}$ we removed only the medial part of the floor in six of 18 patients (11 orbits) who underwent a three wall decompression for moderate exophthalmos. In the other 12, we also resected the lateral part of the lateral floor, but preserved the thin bone overlying the infraorbital nerve.

The postoperative follow up period ranged from 6 to 16 months (mean 9 months).

\section{OPHTHALMIC EXAMINATION}

Before and 2 months after surgery, the amount of proptosis was assessed using a Hertel exophthalmometer. Furthermore, we recorded the (pinhole) visual acuity and intraocular pressure, and performed slit lamp examination and funduscopy. In addition, the visual fields and visual evoked potentials (VEP) were assessed.
ORTHOPTIC EXAMINATION

Orthoptic examination included the assessment of binocular single vision, the cover test (near and distance), the prism cover test (near and distance), the ocular motility, the ocular ductions, and the Hess screen test. These investigations were performed preoperatively and 2 months after surgery. A subdivision into four groups was made: (1) No diplopia (0), (2) diplopia at extremes of gaze (+), (3) occasional diplopia in primary and/or reading position (when tired/in the evening) (++), and (4) continuous diplopia in primary and/or reading position $(+++)$.

\section{PATIENT SATISFACTION}

Patient satisfaction was assessed through a telephonic survey by a medical student (DB). Each patient was asked to score his/her satisfaction with regard to the outcome of surgery on a scale from 1 to 10 ( $1=$ totally dissatisfied; $10=$ excellent $)$. Other questions included the side effects of surgery.

\section{STATISTICAL ANALYSIS}

A paired samples $t$ test was used to calculate the significance of differences between preoperative and postoperative ductions.

\section{SURGICAL TECHNIQUE}

Under general anaesthesia, the lateral canthus and lower eyelid were infiltrated with lignocaine $2 \%$ with $1: 200000$ adrenaline. ${ }^{9} \quad$ A traction suture was placed beneath the insertion of the inferior rectus muscle and the cornea was protected with moistened gel foam. A lateral extended canthotomy of about $15 \mathrm{~mm}$ parallel to the relaxed skin tension lines (Fig 2) was made. The underlying orbicularis muscle and the inferior crus of the lateral canthal ligament were incised, while the eye was pulled upwards using the traction suture. While the lower eyelid was retracted downwards, the lower lid retractors and conjunctiva were cut from the lateral canthus to the caruncle, and prolapsing preseptal orbital fat was resected. The periosteum was incised just anterior of the orbital margin from the level of the caruncle to the lateral canthus and up to the orbital roof. Periosteum elevators and curved retractors were used to dissect the periosteum/ periorbita from the orbital margin and the inferior (Fig 1b and c) and lateral wall (Fig 1d). The lateral wall was visualised after cauterisation and cutting of the zygomaticofacial and zygomaticotemporal arteries and nerves. A hammer and chisel were used to create a bony opening in the lateral wall, while the orbital contents were carefully being kept away with curved retractors. With rongeurs the opening was enlarged in all directions, after blunt dissection of the temporalis muscle with a Freer periosteum elevator. Thick bone was removed by chiselling or burring. With adequate retraction of the temporalis muscle laterally and the orbital contents medially, the deep lateral wall (Fig 1e) was visualised and bone could be removed up to the inner table of the greater wing of the sphenoid. Bone was 

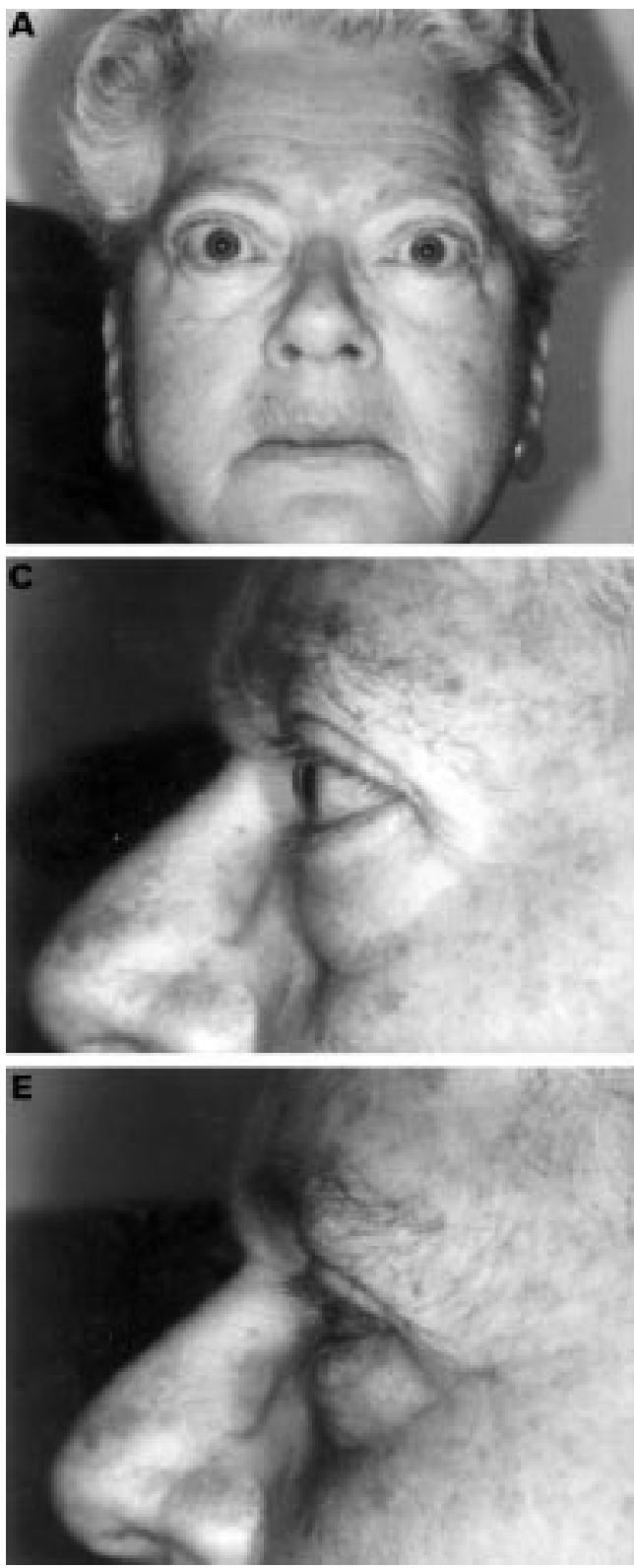

excised as far as the inferior and superior orbital fissures if there was adequate exposure. Any bleeding from diploic vessels was stopped with bone wax.

The orbital floor (Fig $1 \mathrm{~b}$ and c) was removed from approximately $1 \mathrm{~cm}$ posterior to the orbital rim to the end of the maxillary sinus in three wall orbital decompression. A chisel was used to fracture and elevate the bone, and sinus forceps to take bone and maxillary sinus mucosa out. The bone overlying the infraorbital nerve was left intact. The removal of the lateral wall and the floor allowed for better visualisation of the medial wall (Fig 1a). The "orbital strut" (Fig 1 between a and b) - that is, the bony junction of the maxillary sinus and the ethmoid sinus, was left intact to prevent inferomedial displacement. ${ }^{10}$ The medial wall was removed up to the posterior end of the ethmoid sinus. The upper limit of excision was the level of the anterior and posterior ethmoidal arteries (Fig 1 between a and $\mathrm{f}$ ).
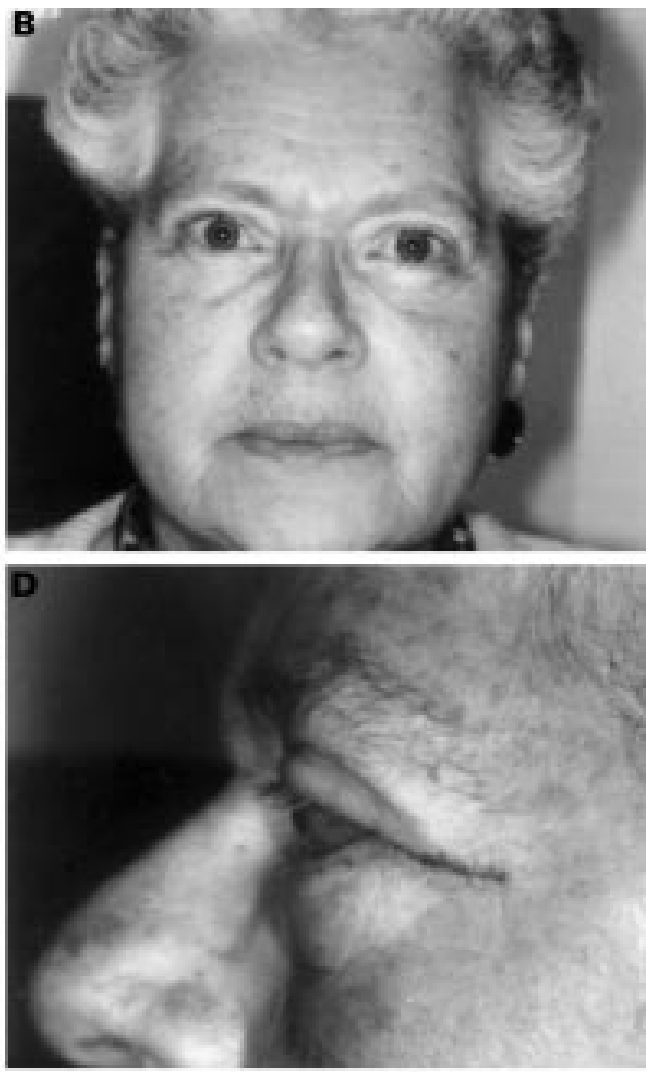

Figure 2 Frontal view showing the preoperative $(A)$ and postoperative appearance after 2 months (B) of a 61 year old woman with burnt out Graves'ophthalmopathy. There was $6 \mathrm{~mm}$ reduction of proptosis after bilateral 2.5 wall orbital decompression at 2 months after surgery. (C) Left lateral view of the same patient depicted in $(A)$ and $(B)$, showing the preoperative appearance $(C)$, and the postoperative appearance after 1 week (D) and after 2 months (E). Note the lateral extended canthotomy scar (on average $15 \mathrm{~mm}$ ) parallel to the relaxed skin tension lines (D).

The lateral, inferior, and medial periorbita were opened with a bent 18 gauge needle. After visible herniation of orbital fat, an Arthro-Lok reverse cutting blade (no 6083, Beaver) was used to cut the periorbita in an anteroposterior direction. ${ }^{9}$ The upper and lower incisions were then joined to allow herniation of orbital fat. This was augmented through blunt dissection with scissors in the spaces between the rectus muscles. The postoperative computed tomography (CT) scans in Figure 3 demonstrate the herniation of orbital tissues in the periorbital spaces following this surgical procedure. Drains were left in the temporal fossa only in case of poor haemostasis. The periosteum and conjunctiva were closed with interrupted absorbable sutures. The lateral canthus was sutured with an absorbable suture (Vicryl 5.0), and the skin was closed with a continuous prolene 6.0 suture.

Postoperative care included antiTrendelenburg position, ice packs, and regular 

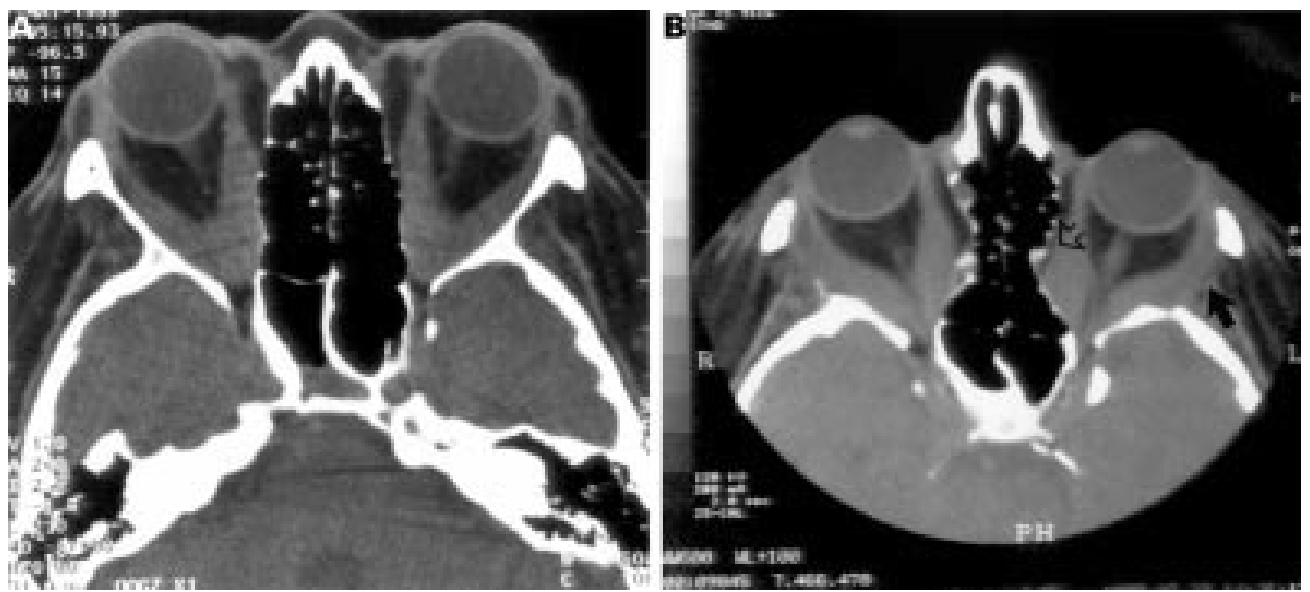

Figure 3 Preoperative axial orbital computed tomography (CT) scan, showing severely enlarged extraocular muscles ("apical crowding") in a case of compressive optic neuropathy (A). The postoperative scan (B) shows the effects of bilateral medial (open arrow) and lateral (closed arrow) wall removal at 2 months after surgery. The visual acuity recovered completely within a week.

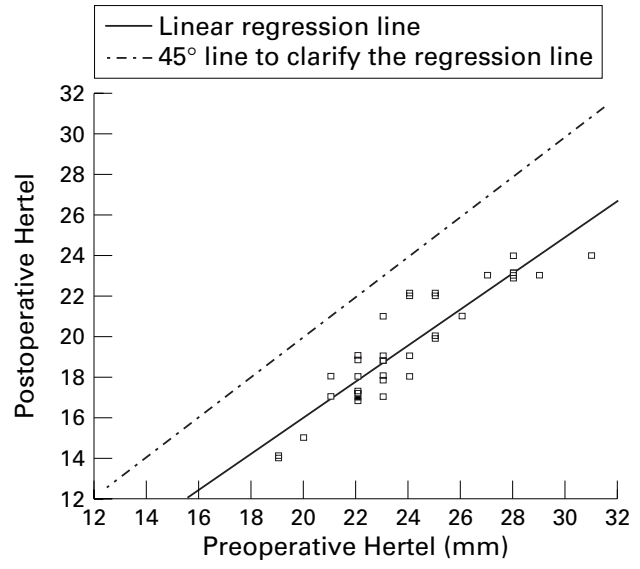

Figure 4 Preoperative and postoperative (at 2 months) Hertel values in 19 patients (35 orbits) who underwent a transconjunctival orbital decompression.

check ups to detect any sudden eye protrusion and persistent bleeding from the orbit or nose. Analgesia (paracetamol/codeine), antiemetic, and antibiotic treatment was given when required.

\section{Results}

PROPTOSIS

In all patients reduction of proptosis was achieved, varying between 3 and $7 \mathrm{~mm}$ (mean $5.5 \mathrm{~mm}$ ) at 2 months following surgery (Fig 4).

\section{VISUAL ACUITY}

The (pinhole) visual acuity of the patients improved shortly after surgery in five of the six patients with compressive optic neuropathy. In the other patient with a combination of severe Graves' ophthalmopathy and diabetes mellitus, there was only gradual improvement of visual function after orbital decompression (within 8 weeks). We found no change of (pinhole) visual acuity in the patients who underwent orbital decompression for rehabilitative reasons.
SIDE EFFECTS AND COMPLICATIONS

The most significant side effect was induced diplopia, which was noted in two out of 19 patients (11\%) (Fig 5). One of these (two) patients had diplopia in extremes of gaze before surgery, the other had no diplopia preoperatively. When we exclude all patients with preoperative continuous diplopia, we calculate the incidence of induced diplopia in this study at $12.5 \%$ (two out of 16 patients). In contrast, reduction of diplopia was seen in three patients $(16 \%)$, while the other 14 (73\%) experienced no change. More specifically, of the eight patients who had no diplopia preoperatively, one patient experienced horizontal diplopia postoperatively (Fig 5). Of the four patients with diplopia at extremes of gaze, diplopia did not change in two patients, while it disappeared in one patient, and worsened in the other. Of the four patients who had diplopia in primary position when tired, two had no diplopia postoperatively and the other two experienced no change. In all three patients who had diplopia continuously before surgery, the degree of diplopia remained unchanged.

The preoperative and postoperative ductions are shown in Figure 6. The mean preoperative and postoperative ductions (in degrees) were 36.7 and 36.9 (abduction), 26.1 and 26.4 (elevation), 38.7 and 39.9 (adduction), and 29.7 and 30.0 (depression) for the right and left eye respectively. None of the ductions changed significantly $(\mathrm{p}>0.05)$. Although in six out of $19(32 \%)$ patients the ductions decreased, diplopia did not change in these patients. In four out of $19(21 \%)$ patients the ductions improved postoperatively; however, two of these patients experienced an increase of diplopia.

Other side effects were postoperative transient conjunctival chemosis $(n=13$ $(81 \%))$, and (transient) hypaesthesia of the infraorbital nerve area $(n=3(19 \%))$. In two cases of unilateral Graves' ophthalmopathy that we treated by three wall decompression, we noted a subtle but visible downward shift of the operated globe 


\begin{tabular}{|c|c|c|c|c|}
\hline Pre- & 0 & + & ++ & +++ \\
\hline 0 & $\mathbf{7}$ & 0 & 1 & 0 \\
\hline+ & 1 & 2 & 0 & 1 \\
\hline++ & 0 & 0 & 2 & 0 \\
\hline+++ & 0 & 0 & 0 & 3 \\
\hline
\end{tabular}

$0=$ no diplopia

$+=$ diplopia at extremes of gaze

$++=$ diplopia in primary or reading position when tired

$+++=$ continuous diplopia in primary or reading position

$\square$ = regression diplopia

$\square=$ progression of diplopia

$\square=$ no change in diplopia

Figure 5. Preoperative and postoperative diplopia in 19 patients who underwent transconjunctival orbital decompression.

("hypoglobus"). One patient had an inferiorly placed lateral canthus following reestablishment of the lateral ligament. Another patient developed a unilateral entropion with secondary keratopathy, which required surgical correction. Finally, one patient developed sinusitis maxillaris. There was no liquor leakage (due to defects in the dura) during or after surgery.
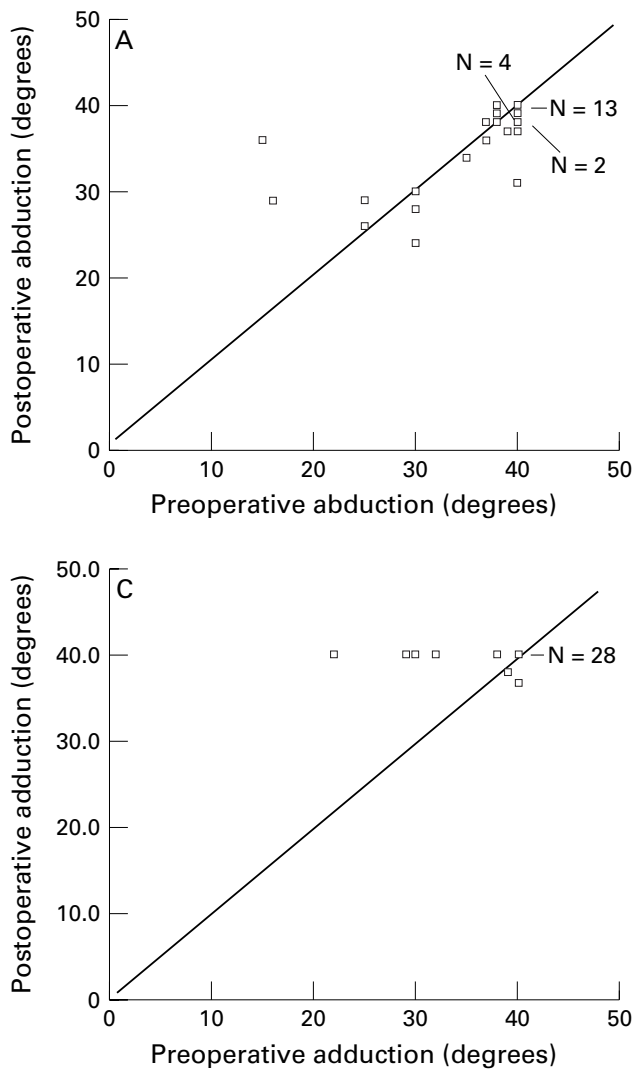

PATIENT SATISFACTION

A telephonic survey among 17 of 19 patients (90\% response rate) revealed general satisfaction towards this method of orbital decompression: the mean general score was 8.2 on a sliding scale from 1 to 10 ( $1=$ totally dissatisfied; $10=$ excellent). This score ranged from 6 to 10 . The lowest score (6) came from a patient with compressive optic neuropathy, who experienced aggraviation of diplopia. Another reason for poor satisfaction was keratopathy as a result of entropion of the lower lid, which complicated the "swinging eyelid" technique. Transient postoperative diplopia was reported by nine (out of 17) patients.

\section{Discussion}

Although the concept of removing bone of the orbital walls to create space for diseased orbital contents may seem illogical, as yet no better means than orbital decompression is available to treat more severe forms of Graves' ophthalmopathy. Improved surgical techniques, using "hidden incisions", and an increased patient intolerance to disease induced physical alterations, have led to a wider use of orbital decompression for cosmetic rehabilitation. ${ }^{812}{ }^{13}$ Each technique holds several side effects, the most prevalent of which is induced diplopia. ${ }^{14}{ }^{15}$ Surgeons have claimed superiority of their method by virtue of lesser complications and lower morbidity. ${ }^{5}{ }^{6}$ However, the reported complication rate of one, single technique varies widely between studies, which hinders comparison between alternate methods of orbital
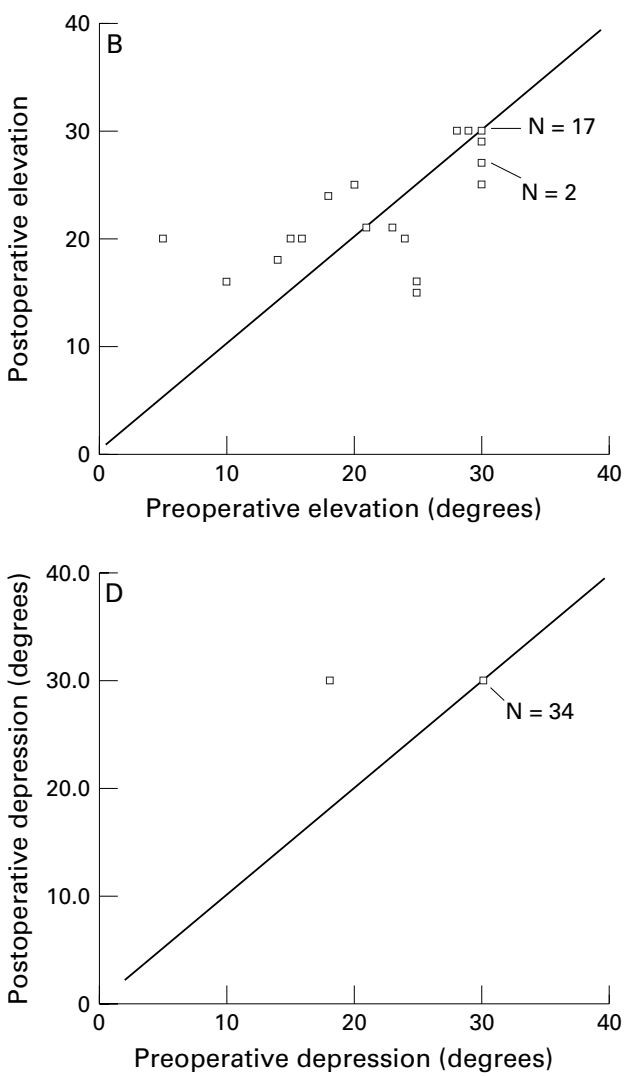

Figure 6 Preoperative and postoperative ductions in 19 patients who underwent transconjunctival orbital decompression. There was no statistically significant change in preoperative and postoperative ductions in any direction $(p>0.05)$. 
decompression. ${ }^{15}$ Recently, ENT surgeons, who were confronted with a high frequency of motility disorders after transantral orbital decompression, ${ }^{45}$ have introduced an endoscopic approach to the orbit, ${ }^{16}$ which unfortunately does not facilitate access to the lateral wall and yields limited reduction of proptosis. This procedure may, however, be combined with another technique to address more advanced cases of Graves' ophthalmopathy. Most orbital surgeons have now abandoned methods that cause visible scars and use hidden incisions, either in the orbital area ${ }^{812}$ or behind the hair line. ${ }^{6}{ }^{13}$

This study indicates that the "swinging eyelid" technique with the inner approach to the lateral orbital wall combines maximal restoration of cosmesis and good functional outcome with minimal morbidity for the patient. It allows the surgeon to address three orbital walls, and, in fact, all bony decompressions may be performed using this method, except when four wall orbital decompression for maximum effect is required. ${ }^{7}$ Also, especially in Asian orbits, a transcaruncular approach to the medial wall may be necessary to provide adequate exposure. ${ }^{9}$ Goldberg et $a l^{17}{ }^{18}$ recently stressed the importance of the lateral wall in orbital decompression surgery. They not only reported that lateral wall removal may result in a significant reduction of proptosis (up to $5 \mathrm{~mm}$ ), but also felt that this surface of decompression is associated with fewer side effects. In postoperative CT imaging (Fig 3), we noted considerable effect of lateral wall removal on the position of the orbital contents, even when the bone of the deep lateral wall had not been entirely drilled up to the dura. In our small series, we obtained an adequate reduction of proptosis in most cases, using a two, a two and a half, or three wall decompression. On the basis of this study, we believe in achieving a "balanced" orbital decompression by lateral and medial wall removal. Only in advanced Graves' ophthalmology, but preferably not in unilateral cases, the orbital floor should be removed, with the "orbital strut" left in place. The floor and "orbital strut" have a supporting role for the orbital contents, and removal of these structures may result in a classic "hypoglobus", which creates not only poor cosmesis but also morbidity, including keratitis and entropion. ${ }^{10}{ }^{11}$ Furthermore, the preservation of supportive orbital structures may reduce the incidence of iatrogenic diplopia..$^{512} 14$ The frequency of induced diplopia at $13 \%$ in our small series was low compared with the transantral decompression method, which has been associated with a frequency between $30 \%$ and $50 \%, 5121415$ and similar to translid and coronal surgical techniques with a $19 \%$ incidence of diplopia. ${ }^{15}$ This study confirms earlier observations that patients who have no complaints of diplopia before orbital decompression are less likely to develop diplopia after surgery, and that those with preoperative diplopia have a high risk of postoperative diplopia. ${ }^{14}$ We observed no significant change of ocular motility after decompression surgery (Fig 6) and noted a discrepancy between diplopia and ocular motility in this study.

Compared with three wall decompression by coronal approach, the transconjunctival technique is not associated with frontalis palsy, forehead anaesthesia, and temporalis atrophy. Furthermore, the postoperative inpatient course is shorter. Both procedures yield good patient satisfaction, ${ }^{15}$ which is important, especially in procedures to improve the patients' cosmetic appearance. Transconjunctival orbital decompression may be efficiently combined with sclera inlay and lower lid blepharoplasty, which otherwise would require separate procedures at a later stage.

The modified removal of the lateral wall ( $a b$ interno) has the following advantages. Firstly, the orbital rim is left intact, thus reducing the postoperative morbidity. Secondly, the temporalis muscle is only minimally disinserted. Disinsertion may lead to cosmetically disfiguring atrophy, and chewing difficulty, as frequently seen after coronal orbital decompression. Thirdly, it is a faster technique when compared with temporary removal of the lateral orbital rim. The disadvantages include: firstly, limited visibility of the deep lateral wall and deep medial wall due to "keyhole surgery"; secondly, the tight working space requires good assistance and surgical skill, especially when total removal up to the dura is required.

In conclusion, the versatile "swinging eyelid" technique is safe and efficacious and may be used for various grades of Graves' ophthalmopathy. Removal of the lateral wall ab interno further adds to minimise the side effects of this operation.

We thank Professor Jack Rootman and Dr Peter Dolman, Vancouver, British Columbia, Canada, for teaching us the basics of the "swinging eyelid" approach. Jessica Leenen is acknowledged for her artistic assistance in producing Figure 1.

1 Dollinger J. Die Druckentlastung der Augenhöhle durch Entfernung der äußeren Orbitalwand bei hochgradigen Exophthalmus (Morbus Basedowii) und konsekutiver Hornhauterkrankung. Dtsch Med Wochenschr 1911;37:1888-90.

2 Olivari N. Transpalpebral decompression of endocrine ophthalmopathy (Graves' disease) by removal of intraorbital fat: experience with 147 operations over 5 years. Plast Reconstr Surg 1991;87:627-41.

3 Linberg JV, Anderson RL. Transorbital decompression: indications and results. Arch Ophthalmol 1981;99:113-9.

4 Walsch TE, Ogura JH. Transantral orbital decompression for malignant exophthalmos. Laryngoscope 1957;67:54468 .

5 McCord CD Jr. Current trends in orbital decompression. Ophthalmology 1985;92:21-33.

6 Mourits MPh, Koornneef L, Wiersinga WM, et al. Orbital Mourits MPh, Koornneef L, Wiersinga WM, et al. Orbital
decompression for Graves' ophthalmopathy by inferomedial, by inferomedial plus lateral, and by coronal approach. Ophthalmology 1990;97:636-41.

7 Kennerdell JS, Maroon JC. An orbital decompression for severe dysthyroid exophthalmos. Ophthalmology 1982;89: $467-72$

8 McCord CD. Orbital decompression for Graves' disease: exposure through lateral canthal and inferior fornix incision. Ophthalmology 1981;88:533-41.

9 Rootman J, Stewart B, Goldberg RA. Decompression for thyroid orbitopathy. In: Orbital surgery: a conceptual approach. Philadelphia: Lippincott-Raven, 1995:353-84.

10 Bosch WA van den, Tjon-Fo-Sang $M$, Lemij HG. Eyeball position in Graves' orbitopathy, and its significance for eyelid surgery. Ophthalmic Plast Reconstr Surg 1998;14:

11 Goldberg RA, Shorr N, Cohen MS. The medial orbital strut in the prevention of postdecompression dystopia in dysthyroid ophthalmopathy. Ophthalmol Plast Reconstr Surg 1992; 8:32-4. 
12 Lyons CJ, Rootman J. Orbital decompression for disfiguring exophthalmos in thyroid orbitopathy. Ophthalmology 1994; 101:223-30

13 Krastinova D, Rodallec A. Orbitopathie Basedowienne. Ann Chir Plast Esthet 1985;30:351-8.

14 Shorr N, Neuhaus RW, Baylis HI. Ocular motility problems after orbital decompression for dysthyroid ophthalmopathy. Ophthalmology 1982;89:323-8.

15 Paridaens D, Hans K, van Buitenen S, et al. The incidence of diplopia following coronal and translid orbital decompression in Graves' orbitopathy. Eye 1998;12:800-5.
16 Neugebauer A, Nishimo K, Neugebauer P, et al. Effects of bilateral orbital decompression by an endoscopic endonasal approach in dysthyroid orbitopathy. $\mathrm{Br} \mathcal{F}$ Ophthalmol

7 Goldberg RA. The evolving paradigm of orbital decompression surgery. (Editorial) Arch Ophthalmol 1998;116: 95-6.

18 Goldberg RA. The lacrimal keyhole, orbital door jamb, and basin of the inferior orbital fissure. Three areas of deep bone in the lateral orbit. Arch Ophthalmol 1998;116:161824. 